\title{
Absorption Measurement Errors in Single-Mode Fibers Resulting from Re-Emission of Radiation
}

\author{
Yutong Feng, Betty Meng Zhang, Junqing Zhao, Sheng Zhu, \\ Jonathan H. V. Price, and Johan Nilsson
}

\begin{abstract}
We investigate errors in small-signal absorption spectra that result from re-emission in single-mode fibers with overlapping absorption and emission spectra. Experiments on Er-doped fibers and simulations of Er and Yb-doped fibers show that the re-emission can severely distort the spectrum, especially the peak, under common measurement conditions, and underestimate the absorption by well over $10 \%$ already at $30 \mathrm{~dB}$ peak absorption, if only the source or the detector is spectrally filtered. Re-emission can then be the dominant source of errors. The error increases for higher absorption and higher fiber-NA. For sufficiently high NA, a significant error remains even in the limit of zero absorption and reaches $5 \%$ at the peak of a $0.46-\mathrm{NA}$ Yb-doped fiber. Furthermore, in contrast to the high-absorption case, the error is larger at longer wavelengths than at the peak. Simultaneous filtering of both source and detector with $0.1 \mathrm{~nm}$ bandwidth reduces the re-emission error to $\sim 1 \%$ or less up to 90 dB absorption. Then, detector noise or saturation errors are likely to dominate and render re-emission errors insignificant. A standard amplifier model is well suited to the simulations of the rich physics of single-mode-fiber absorption measurements.
\end{abstract}

Index Terms-Absorption, Fiber characterization, Spectroscopy

\section{INTRODUCTION}

A bsorption and emission of electromagnetic radiation are key properties of any laser medium and the subject of continuous and current interest, notably in fibers [1], [2], [3], [4], [5], [6]. The absorption spectrum, and in particular its peak value, is often the first measurement on an optical fiber, and the result then influences future fabrication as well as the laser design.

Following absorption, light can be spontaneously re-emitted at a different frequency and propagate in any direction. As a result, the spectrum of the light changes. This process was studied in sodium vapor in the early twentieth century [7] and its impact on absorption characterization was later studied in various atomic vapors [8], [9]. The process can be cascaded and is particularly strong at the high levels of absorption in

Manuscript received March 16, 2017; revised April 10, 2017; accepted April 18, 2017. This work was supported in part by the EPSRC (FLITES, EP/J00278X/1 and the EPSRC Centre for Innovative Manufacturing in Photonics, EP/H02607X/1) and in part by AFOSR (FA9550-14-1-0382).

Yutong Feng, Sheng Zhu, Jonathan H. V. Price and Johan Nilsson are with Optoelectronics Research Centre, University of Southampton, Southampton SO17 1BJ, UK. (e-mail:yf1n11@soton.ac.uk; sz4g14@soton.ac.uk; jhvp@orc.soton.ac.uk; jn@orc.soton.ac.uk).

Betty Meng Zhang is now Visiting Academic with Optoelectronics Research Centre, University of Southampton, Southampton SO17 1BJ, UK. (e-mail: bettyzhanglab@gmail.com)

Junqing Zhao is now with the Jiangsu Key Laboratory of Advanced Laser Materials and Devices, School of Physics and Electronic Engineering, Jiangsu Normal University, Xuzhou 221116, China. (junqingzhao@outlook.com). optically dense media. Thus, whereas it is conceptually straightforward to measure the transmission of a light probe through a laser medium, and to calculate the absorption from that, several factors can impair the accuracy and the interpretation of the data. It is for example well-known that if the probe power is excessive, the excitation of the laser medium can become large enough to modify the size and spectral shape of the absorption as a result of a reduced population in the absorbing ground state and stimulated emission from excited states. The spectrum then deviates from the small-signal absorption spectrum as obtained in the absence of an excited population. The measured spectrum may also be distorted by nonlinear wavelength conversion, e.g., if a supercontinuum (SC) source with high peak power is used for the measurements. Normally the more fundamental smallsignal absorption is the primary interest, and relative to that, these distortions constitute measurement errors. Such errors are particularly likely to occur in a fiber, because tight confinement of power increases any nonlinear wavelength conversion and saturation and because a long interaction length further promotes nonlinear conversion. Fibers with high absorption require high probe powers in order to reach detectable transmitted power levels, and are therefore particularly problematic to characterize.

In addition, in multimode structures such as double-clad fibers, differences in the small-signal absorption between different modes can also modify the absorption spectrum [2], [3]. This is not a measurement error but rather a true effect that reduces the absorption in longer fibers due to different overlap of different pump modes with the doped region and imperfect mode mixing. This typically reduces the efficiency and / or necessitates the use of even longer fibers. Also these effects are especially strong for high absorption values, and therefore they have strongest effect for the absorption peak.

Still, apart from our recent concise conference paper [10], there has not been any work on how the spontaneous reemission of absorbed light perturbs the transmission through fibers doped with laser-ions and thus the characterization of absorption spectra. This happens also in single-mode fibers, and is the subject of this paper.

Specifically, we investigate measurement errors in continuous-wave small-signal absorption spectra in singlemode fibers resulting from spontaneous re-emission of absorbed light at wavelengths that overlap with the absorption line under study. Since the re-emission adds to the power detected at the fiber output, it leads to an underestimate of the absorption. Although the distorted spectrum perhaps can be 
viewed as an absorption spectrum, too, Beer's law no longer applies and it deviates from the small-signal absorption spectrum as determined by the absorption cross-sections and the overlap factor. Therefore we view also these distortions as errors. This is further motivated by the fact that the reemission does not influence the absorption characteristics of pump or signal in a laser, and therefore do not directly influence the characteristics of an operating laser. However given the importance of the value of the absorption spectrum and peak absorption for device modeling and optimization, an error in this may well lead to non-optimal designs, especially when experimental optimization is difficult.

The re-emission error is a linear effect which occurs also at low powers, since each absorbed photon excites an ion, which can then re-emit. We experiment on an Er-doped fiber and use simulations with a standard amplifier model to further investigate Er- and Yb-doped fibers. Experiments and simulations agree well. The error becomes more important when a large fraction of the re-emitted light is captured by a fiber with high NA, when the laser medium has a distinct absorption peak on a broad shoulder, and when the required wavelength-selective filtering occurs at either the source or the detector end of the measurement setup, but not at both ends.

There are two regimes: For the-low absorption regime, the probe photons absorbed by the dopant can to a significant fraction be compensated for by re-emitted photons. This leads to an error of $5 \%$ at the absorption peak in an Yb-doped fiber (YDF) with NA $=0.46$ in the limit of zero absorption. Furthermore, the relative error becomes larger for longer wavelengths. This is in contrast to the other, high-absorption regime, which can lead to larger errors and is the regime we primarily consider. At high absorptions, the few photons that reach the detector can be dominated by re-emitted photons rather than by the directly transmitted "ballistic" probe photons which correspond to the actual small-signal absorption. This affects the absorption peak most strongly, and more so the higher the peak absorption. It is thus another effect that also leads to the often-seen reduction of the measured peak absorption at high absorption levels. This is frequently attributed to differential mode absorption in multimode fibers, but the use of single-mode fibers eliminates this in our investigations. Simulations show that the error in peak absorption can reach $5 \%$ already at $20 \mathrm{~dB}$ peak absorption, and well over $10 \%$ at $30 \mathrm{~dB}$ peak absorption, when measured with a white-light source in a normal single-mode fiber. With the same configuration, at $60 \mathrm{~dB}$ actual peak absorption, the apparent peak absorption (as determined directly from the output spectrum) can be as low as $30 \mathrm{~dB}$. Reemission can then be the dominant and unacceptable source of error. On the other hand, if both the source and the detector are spectrally filtered with $0.1 \mathrm{~nm}$ bandwidth, then the reemission error becomes as low as around 1\% even for as much as $90 \mathrm{~dB}$ absorption. At such high absorption, other sources of error such as detector noise and saturation effects are likely to be more severe. Thus, with this more refined measurement setup, it is possible to eliminate re-emission as a significant source of error.
Though some simulations use extreme or unusual parameters to illustrate a point, others correspond to parameters and situations often encountered in practice. For example, $30 \mathrm{~dB}$ measurement range is readily achievable with a tungsten filament light source and a conventional optical spectrum analyzer (OSA). This makes it is easy to fall into the trap of measuring too high absorption and thus introduce reemission errors. The increasing use of SC sources with their greater measurement range can lead to even larger errors.

\section{CONFIGURATION}

Fig. 1 illustrates the configuration we used for experiments and primarily consider for simulations. It is a conventional setup for fiber absorption measurements. Experimentally, we have a choice between a white-light source (WLS, in our case a Fianium FM1060-40 / PA1060-SC supercontinuum source) or a tunable laser source (TLS, Agilent 81640A), as well as between an Ando AQ6317B OSA and an InGaAs broadband power meter (HP 8153A lightwave multimeter with HP 81533B interface module and HP 81525A optical head). We used the WLS and the OSA for most measurements, including all measurements of absorption spectra, with the OSA set to 1 $\mathrm{nm}$ resolution. The detected signal was in every case unaffected by receiver noise, so that clean traces were obtained. Furthermore we used the conventional fiber cutback approach to eliminate splice-loss errors. A dichroic mirror and a 25-km long standard single-mode fiber removed wavelengths shorter than $1100 \mathrm{~nm}$ from the SC spectrum. Fig. 2 shows the spectra of the TLS (tuned to $1529.5 \mathrm{~nm}$ ) and the SC source, as launched into the fiber under test. The total launched power was $100 \mu \mathrm{W}$ from the TLS and $20 \mu \mathrm{W}$ from the SC-source. The SC power density was $\sim 100 \mathrm{nW} / \mathrm{nm}$ across the Er-absorption band, but dropped by $\sim 5 \mathrm{~dB}$ at the short end of the band at $\sim 1425 \mathrm{~nm}$.

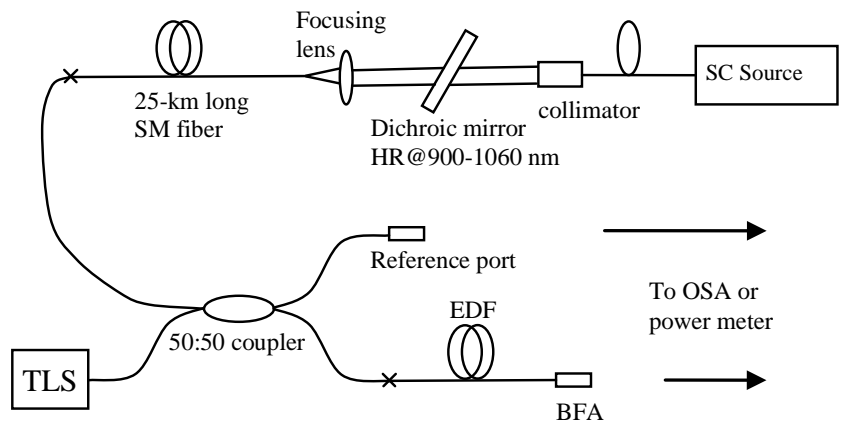

Fig. 1. Absorption measurement setup. BFA: bare fiber adaptor. See text for other abbreviations. 


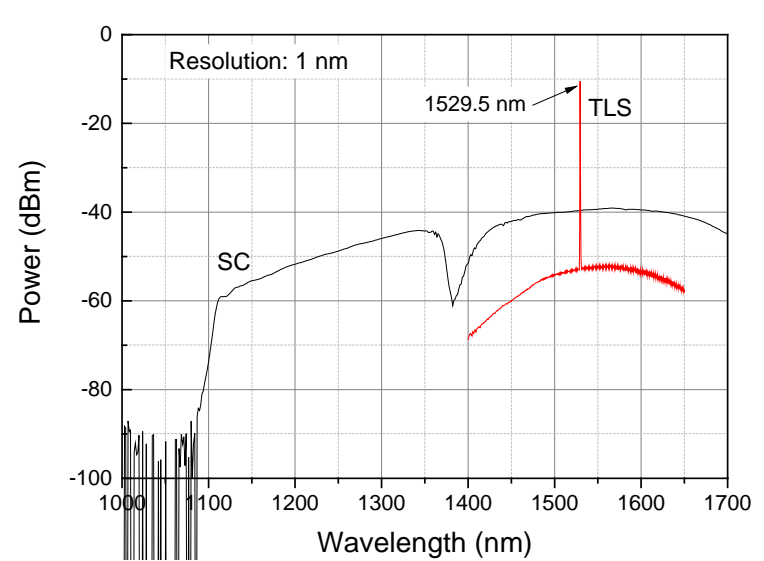

Fig. 2. Spectrum of SC-source and TLS as launched into the EDF.

The fiber tested experimentally was a single-mode Er-doped fiber (EDF, Fibercore I-12(980/125) HC). It has an NA of 0.25 and a mode-field diameter of $5.13 \mu \mathrm{m}$, as characterized by the manufacturer. The peak absorption at $\sim 1530 \mathrm{~nm}$ is $21 \mathrm{~dB} / \mathrm{m}$. The ${ }^{4} I_{13 / 2}$-level $(\sim 1.5 \mu \mathrm{m})$ was the only erbium-level that absorbed light, because of the absence of probe light at wavelengths of $980 \mathrm{~nm}$ or shorter. We calculate the saturation power at the absorption peak to $230 \mu \mathrm{W}$.

For all simulations with WLS probing, the input had a uniform spectral power density of $1 \mathrm{nW} / \mathrm{nm}$ across the absorption band under study (1400 nm - $1619.8 \mathrm{~nm}$ for Er and $850-1140 \mathrm{~nm}$ for $\mathrm{Yb}$ ) and zero power outside. In case of the TLS, the power was $1 \mathrm{nW}$ within the narrow linewidth of the probe and zero elsewhere. Whereas TLS measurements normally use higher power than $1 \mathrm{nW}$, the linearity of reemission errors makes this unimportant for our simulations (which were free from noise). Also the OSA was assumed to be ideal in the simulations, without any leakage of out-of-band light. All plotted output spectra display the power in $1 \mathrm{~nm}$.

Fig. 3 shows the Er and Yb cross-section spectra we used. They are taken from RP Fiber Power v. 5 [11], [12], which is the software we used for all simulations. The Er cross-sections are for an aluminosilicate host. We adjusted the Erconcentration to $9.741 \times 10^{24}$ ions $/ \mathrm{m}^{3}$ in order to match the measured peak absorption, and found that also the shape of the absorption spectrum matched measured data. The emission cross-section is set to zero for wavelengths below $1450 \mathrm{~nm}$. Also, both the emission and absorption cross-sections are truncated by RP Fiber Power at $1619.8 \mathrm{~nm}$, since excited-state absorption makes longer wavelengths irrelevant for simulations of amplification in aluminosilicate EDFs. For the Yb-doped fibers, we used a concentration of $5 \times 10^{24}$ ions $/ \mathrm{m}^{3}$, which leads to a peak absorption of $42 \mathrm{~dB} / \mathrm{m}$. Although we simulated fibers of different NA, they are all single-mode step-index fibers with $V=2$ at $1529 \mathrm{~nm}$ (Er) and $975 \mathrm{~nm}(\mathrm{Yb})$.

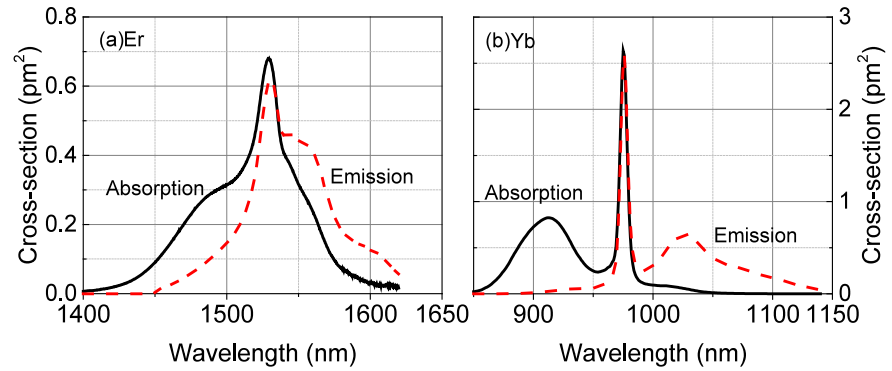

Fig. 3. Cross-section spectra used in simulations, (a) Er:aluminosilcate, (b) Yb:germanosilicate. From [11], [12].

\section{DESCRIPTION OF THE MODEL USED FOR SIMULATIONS}

We used standard equations as implemented in RP Fiber Power for a single-mode fiber amplifier with homogeneously broadened absorption and emission cross-sections and radially resolved modal fields and dopant excitations to evaluate how the spectra of probe and re-emitted light evolve in a fiber:

$$
\begin{aligned}
d S_{P}(\lambda, z) / d z & =\left[\gamma_{e}(\lambda, z)-\gamma_{a}(\lambda, z)\right] \cdot S_{P}(\lambda, z) \\
d S_{A S E}(\lambda, z) / d z & =2 h c^{2} \gamma_{e}(\lambda, z) / \lambda^{3} \\
& +\left[\gamma_{e}(\lambda, z)-\gamma_{a}(\lambda, z)\right] \cdot S_{A S E}(\lambda, z)
\end{aligned}
$$

Here, $S_{P}$ is the spectral power density of the probe, which can be spectrally broad or narrow, and $S_{A S E}$ is the spectral power density (power per unit wavelength) of the spontaneous emission (in an amplifier this becomes amplified spontaneous emission, but the lack of pumping means the net gain is negative). Furthermore, $h$ is Planck's constant, $c$ is the speed of light, $\lambda$ is the wavelength, and $z$ is the longitudinal coordinate along the direction of propagation. The gain and absorption coefficients $\gamma_{e}$ and $\gamma_{a}$ are given by

$$
\begin{aligned}
& \gamma_{e}(\lambda, z)=\sigma_{e}(\lambda) \cdot 2 \pi \int_{0}^{b} N_{2}(r, z) I(r, \lambda) r d r \\
& \gamma_{a}(\lambda, z)=\sigma_{a}(\lambda) \cdot 2 \pi \int_{0}^{b} N_{1}(r, z) I(r, \lambda) r d r
\end{aligned}
$$

where $\sigma_{e}$ and $\sigma_{a}$ are the emission and absorption crosssections, $b$ is the dopant radius, $N_{1}(r, z)$ and $N_{2}(r, z)$ are the population densities in the ground and excited state, respectively, and $I(r, \lambda)$ is the normalized mode intensity distribution. $N_{2}(r, z)$ can be determined from the rates for stimulated emission $W$ and absorption $R$,

$$
R(r, z)=\int \sigma_{a}(\lambda) I(r, \lambda)\left(S_{P}(\lambda)+S_{A S E}(\lambda)\right) \lambda /(h c) d \lambda
$$

$W$ is given by the same equation with $\sigma_{a}$ replaced by $\sigma_{e}$. Then,

$$
\begin{aligned}
N_{2}(r, z) & =N_{0} R(r, z) /\left(R(r, z)+W(r, z)+\tau^{-1}\right) \\
& \approx N_{0} R(r, z) \tau
\end{aligned}
$$

where $\tau$ is the fluorescence lifetime and $N_{0}$ is the concentration of laser ions. In our simulations we assume that the dopants are uniformly distributed and that $N_{1}+N_{2}=N_{0}$. The approximation in (6) is valid when $R+W<<\tau^{-1}$. This generally applies in our case, because the power is low. 
Equation (1) for the probe spectrum can be integrated and the solution written in terms of the gain $G_{d B}(\lambda) \equiv 10 \log _{10}\left(S_{P}\right.$ $\left.(\lambda, L) / S_{P}(\lambda, 0)\right)$ as

$$
\begin{aligned}
G_{d B}(\lambda)= & 10 \log _{10}(e) \cdot \Gamma(\lambda) L N_{0} \\
& \cdot\left[\left(\sigma_{e}(\lambda)+\sigma_{a}(\lambda)\right) n_{2}-\sigma_{a}(\lambda)\right]
\end{aligned}
$$

Here, $\Gamma(\lambda)$ is the overlap integral between the mode and doped region, $L$ is the fiber length, and $n_{2}$ is the fractional excitation of the rare earth ions, as averaged longitudinally and transversally over the core. We neglect the impact of excited-state absorption, since the low optical power leads to a small excited state population (small $n_{2}$ ). Also, when $n_{2}$ is much smaller than $1+\sigma_{e}(\lambda) / \sigma_{a}(\lambda)$, it can be neglected in (7) to yield the small-signal absorption spectrum $\alpha(\lambda)$ (in decibels) as obtained in the absence of excited ions:

$$
\begin{aligned}
\alpha(\lambda) & =10 \log _{10}(e) \cdot \Gamma(\lambda) L N_{0} \sigma_{a}(\lambda) \\
& =-10 \log _{10}\left(S_{P}(\lambda, L) / S_{P}(\lambda, 0)\right)
\end{aligned}
$$

This equals $-G_{d B}(\lambda)$ when $n_{2}$ is negligible and can be used as a definition of the small-signal absorption spectrum $\alpha(\lambda)$. This is thus proportional to the fiber length according to Beer's law.

On the other hand, even when $n_{2}$ is sufficiently small to be negligible in (7), it is not zero in the presence of probe light and is not negligible in the solution of (2). This allows emitted photons $\left(S_{A S E}\right)$ to build up in the guided mode according to (2), for which there is no solution as simple as that for the probe ((7) and (8)). Insofar as $S_{P}$ and $S_{A S E}$ are not separately measured, the presence of emitted photons (i.e., fluorescence) in the measured output spectrum distorts the determination of $\alpha(\lambda)$ according to (8). Since at low power levels, $n_{2}$ is proportional to the spectral power density of the probe $S_{P}$, also $S_{A S E}$ is proportional thereto. Therefore, at low power levels, the distortion is independent of the probe power.

We used (8) to directly calculate the actual absorption spectrum and $S_{P}$. We also solved numerically (1) - (6) with appropriate boundary conditions, to yield $S_{A S E}$ as well as $S_{P}$. For this, we used RP Fiber Power in all cases. In case of a WLS with spectrally resolved detection, the apparent absorption spectrum $\alpha_{W L S}(\lambda)$, which corresponds to the measured spectrum, can then be calculated according to (9):

$$
\alpha_{W L S}=-10 \log _{10}\left(\left(S_{P}(\lambda, L)+S_{A S E}(\lambda, L)\right) / S_{P}(\lambda, 0)\right)
$$

In case of TLS + broadband detection, the apparent absorption at the wavelength of the TLS is determined from the ratio of the total output power $P(L)$ to the total input power $P(0)$ :

$$
\alpha_{T L S}=-10 \log _{10}(P(L) / P(0))
$$

We also mention that RP Fiber Power actually treats bidirectional propagation equations and considers emission into the forward as well as backward direction (through an equation similar to (2)) as well as Rayleigh back-scattering. However, we found the impact of backward-propagating light to be negligible. Thus, the propagating spectrum at each point inside a fiber can be taken as the output spectrum for the corresponding fiber length. This is in contrast to a typical amplifier, for which coupling between forward and backwardpropagating light is significant.

\section{RESULTS AND DISCUSSION}

Fig. 4 shows how the experimentally measured (apparent) peak absorption, as determined from the light entering into, and exiting from, the EDF under test, depends on the fiber length for three different measurement methods. Best is to use the TLS combined with the OSA, to achieve spectral selection at both source and detector. In this case, the measured absorption is proportional to fiber length over the entire measurement range up to a peak absorption of $58 \mathrm{~dB}$. By contrast, with a WLS instead of the TLS, the measured absorption reaches only $33 \mathrm{~dB}$, whereas the actual value at 2.8 $\mathrm{m}$ is $75 \%$ higher. With the TLS combined with the broadband power meter, the measured absorption only reaches $21 \mathrm{~dB}$.

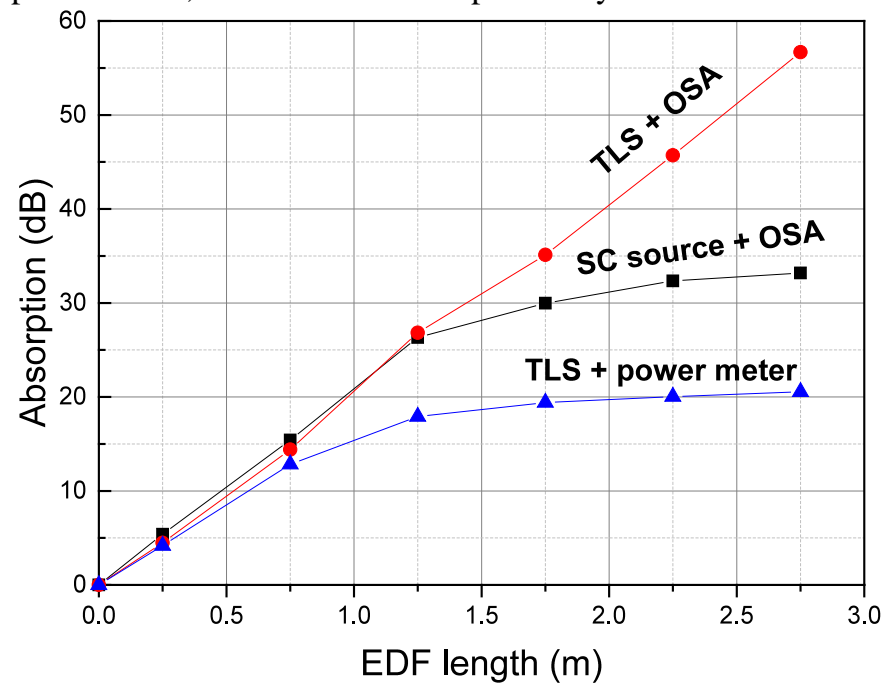

Fig. 4. Measured (apparent) absorption at $1530 \mathrm{~nm}$ vs. length of the Fibercore EDF measured with three different methods.

Fig. 5 shows measured and simulated apparent absorption spectra as determined through cutback for different EDF lengths. An 11-cm length provided the reference spectrum in the cutback calculations for both measurements and simulations. The simulated EDF had an NA of 0.242 and a mode-field diameter of $4.93 \mu \mathrm{m}$, in good agreement with the experimental values. 


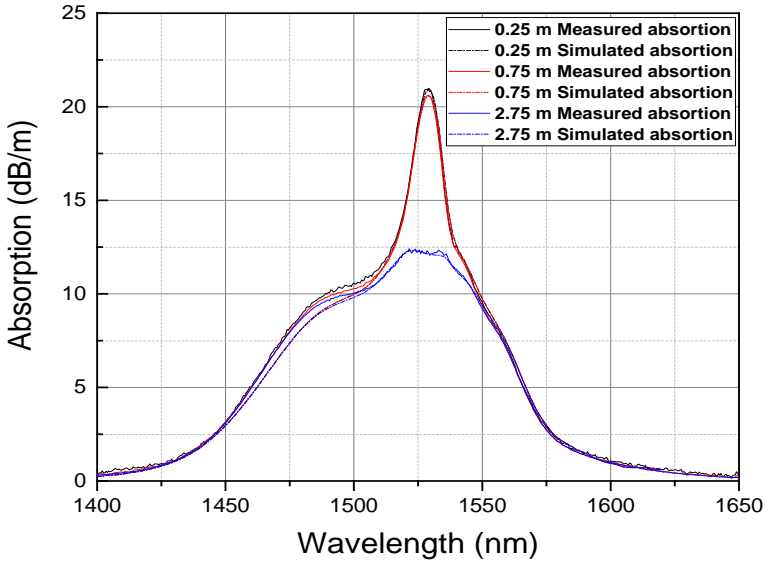

Fig. 5. Apparent absorption spectra per unit length determined with WLS \& OSA for three different EDF cutback lengths with NA $=0.242$. The experimental and simulated curves agree very well and are difficult to distinguish. In addition, the curves for $0.25 \mathrm{~m}$ and $0.75 \mathrm{~m}$ are quite close and also difficult to distinguish.

The near-complete elimination of the absorption peak with minimal distortion away from the peak is striking, as is the excellent agreement between measurements and simulations. The spectral characteristics cannot be explained by wellknown saturation effects (which make the Er-excitation nonnegligible in (7)) since the power of the SC source is much smaller than the saturation power. Furthermore, the lengthdependence and spectral distortions caused by saturation would be qualitatively different.

Rather, we attribute the distortion and the reduction in measured absorption to photons that are wavelength-converted in the fiber. In case of a WLS, a significant number of photons launched into the fiber at low-absorbing wavelengths can propagate through a large fraction of the fiber before they are absorbed. They are then re-emitted at the absorption peak with a probability that is relatively high, since the peak of the fluorescence spectrum overlaps that of the absorption. A fraction of the re-emitted photons couple into the forwardpropagating mode, and although the absorption is high at this wavelength, they are now closer to the detector and still reach it in significant numbers. At the absorption peak, such reemitted photons can then dominate over the few probephotons that travel through the fiber without absorption. This distorts the spectrum. As explained in Sect. III, the spectral distortions are power-independent as long as the excitation of Er-ions is small.

A corresponding process occurs in case of a TLS combined with a broadband detector. Note however that the TLS spectrum in Fig. 2 shows significant background power extending to wavelengths where the Er-absorption is small. This adds to the transmitted power as measured by the broadband power meter and actually dominates the error at high absorption in Fig. 4 for that configuration. Also, the relatively poor spectral selectivity of the TLS combined with a small amount of saturation at $100 \mu \mathrm{W}$ of launched power explains at least in part why the low-absorption values are different with that configuration.

Further simulations of similar and different fibers provide further insight and substantiate our explanation of the distortions. We first consider the same EDF as that used in Fig. 4 \& 5 as well as another one with half the NA and twice the core diameter (so with the same V-value), with WLS probing. Fig. 6 shows the resulting simulated output spectra. For short fibers, $S_{P}$ dominates over $S_{A S E}$, whereas for the 3-m long fiber, $S_{A S E}$ is larger than $S_{P}$ for the wavelength range of 1519.1 - $1538.1 \mathrm{~nm}(\mathrm{NA}=0.121)$ and $1514.6-1545.3 \mathrm{~nm}$ $(\mathrm{NA}=0.242)$. These ranges include the absorption peak. Thus, the fluorescence makes the apparent absorption much smaller than the actual absorption. We see also that the fluorescence power increases by $6 \mathrm{~dB}$ (i.e., four times) when the NA is doubled. This makes the errors larger. The absence of fluorescence at wavelengths shorter than $1450 \mathrm{~nm}$ is an artefact of the truncation of the emission cross-section spectrum. However the fluorescence power in the truncated range is negligible, since the probe power is over $30 \mathrm{~dB}$ larger.

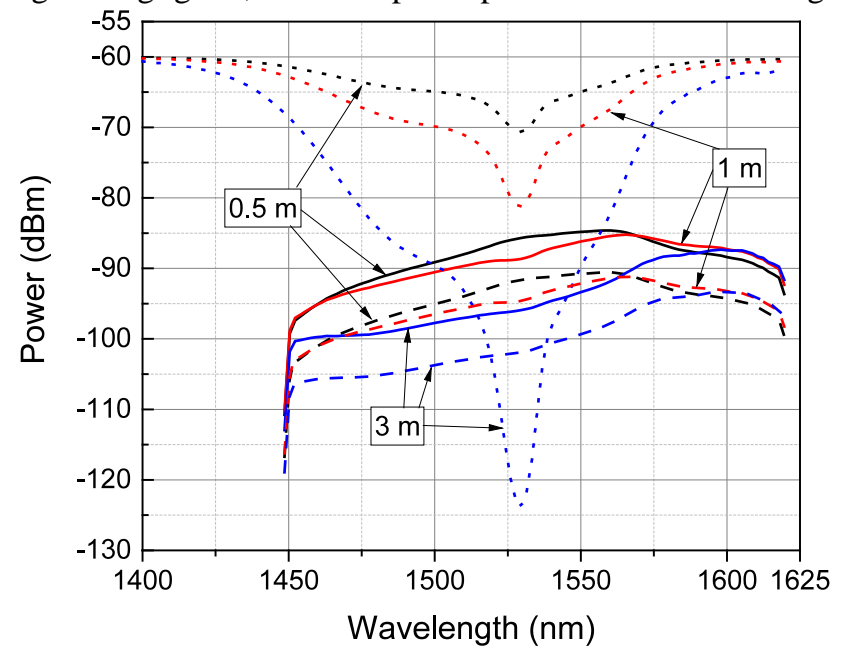

Fig. 6. Output spectra with WLS probe for Er-doped fiber with 0.242 and 0.121 NA. Fiber lengths $0.5 \mathrm{~m}$ (black curves), $1 \mathrm{~m}$ (red curves), and $3 \mathrm{~m}$ (blue curves). Solid curves: fluorescence for NA $=0.24$. Dashed curves: fluorescence for NA $=0.121$. Dotted curves: probe (same for both NAs).

Fig. 7 shows how the power at the 1529-nm absorption peak as well as at 1460, 1550, and $1600 \mathrm{~nm}$ evolve along the two EDFs with the two different NAs. The NA is clearly important. At the peak, the spectral power density of the fluorescence exceeds that of the probe for fibers longer than $1.82 \mathrm{~m}$ (absorption $38.3 \mathrm{~dB}$ ) for $\mathrm{NA}=0.121$, or $1.47 \mathrm{~m}$ (absorption $30.9 \mathrm{~dB}$ ) for $\mathrm{NA}=0.242$. The probe power decreases faster than the fluorescence power in all cases, and especially at the peak in long fibers. It would be challenging to measure a probe power as low as $-165 \mathrm{dBm}$ (0.24 photons per second), but it is possible to increase the WLS spectral power density by a factor of 2000 (to $2 \mu \mathrm{W} / \mathrm{nm}$ ) within acceptable levels of saturation, and the resolution bandwidth by a factor of 5 , and thus increase the received power by $40 \mathrm{~dB}$. A typical OSA can measure down to $-90 \mathrm{dBm}$. With standard instrumentation it is therefore possible to measure down to $130 \mathrm{dBm}$ if these values are rescaled to the conditions of Fig. 6 and 7. In any case, even when the power levels are extreme and too weak to measure, the graphs help to illustrate the different evolutions of probe and fluorescence. 

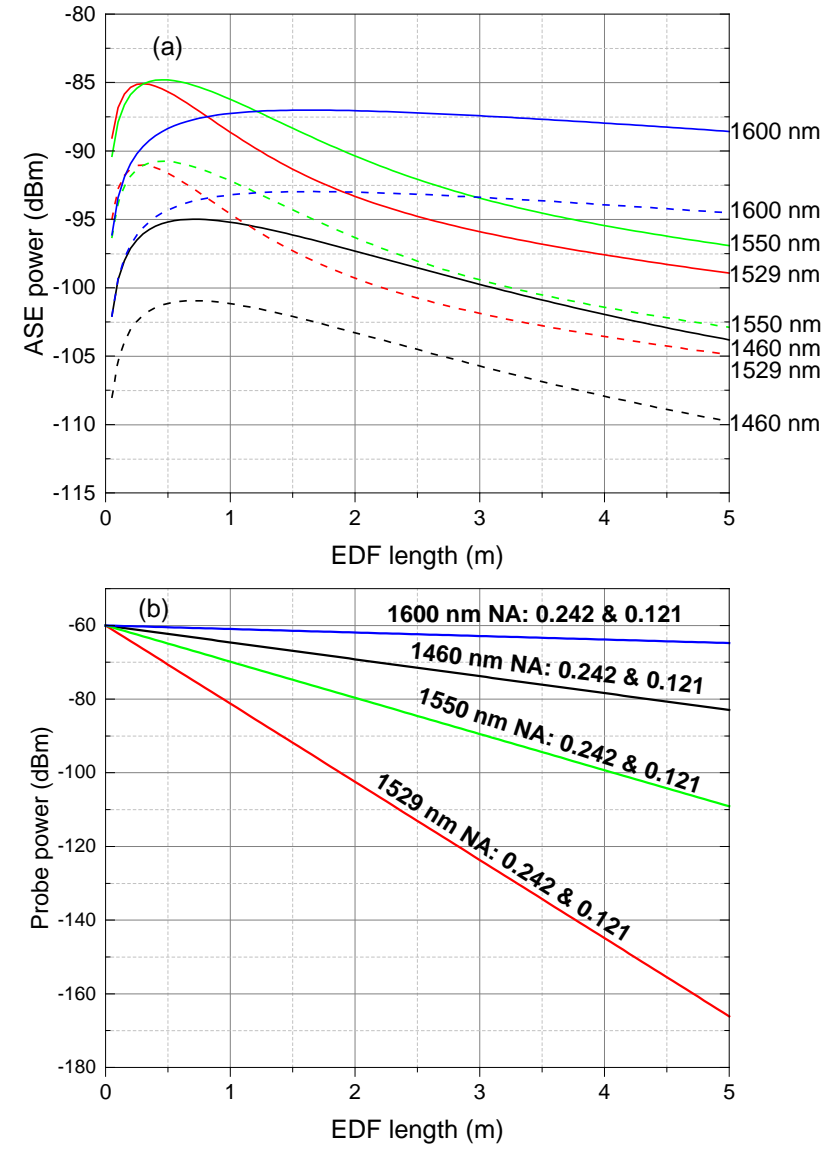

Fig. 7. Evolution of power within $1 \mathrm{~nm}$ along EDFs with NA 0.242 and 0.121 at different wavelengths, (a) fluorescence power ( $\mathrm{NA}=0.242$ : solid curve; NA = 0.121: dashed curve), (b) probe power. Note difference in power axis scales.

Fig. 8 shows the error in the peak absorption in case of WLS probing for the fibers in Fig. $6 \& 7$ as well as some additional Er- and Yb-doped fibers, all with $V=2$. For an EDF with an NA of 0.242, the error with a white light source in Fig. $8(a)$ is $39 \%$ at an actual absorption of $58 \mathrm{~dB}$. This agrees well with the measurements in Fig. 4. Furthermore, Fig. 8(c) shows that the apparent peak absorption grows only very slowly at values beyond between 20 and $40 \mathrm{~dB}$ for the fibers we investigated. Thus, in case of a $0.242-\mathrm{NA} E D F$, if the measured (apparent) peak absorption is $35 \mathrm{~dB}$, the error can be very large (this is also clear from Fig. 8(b)).
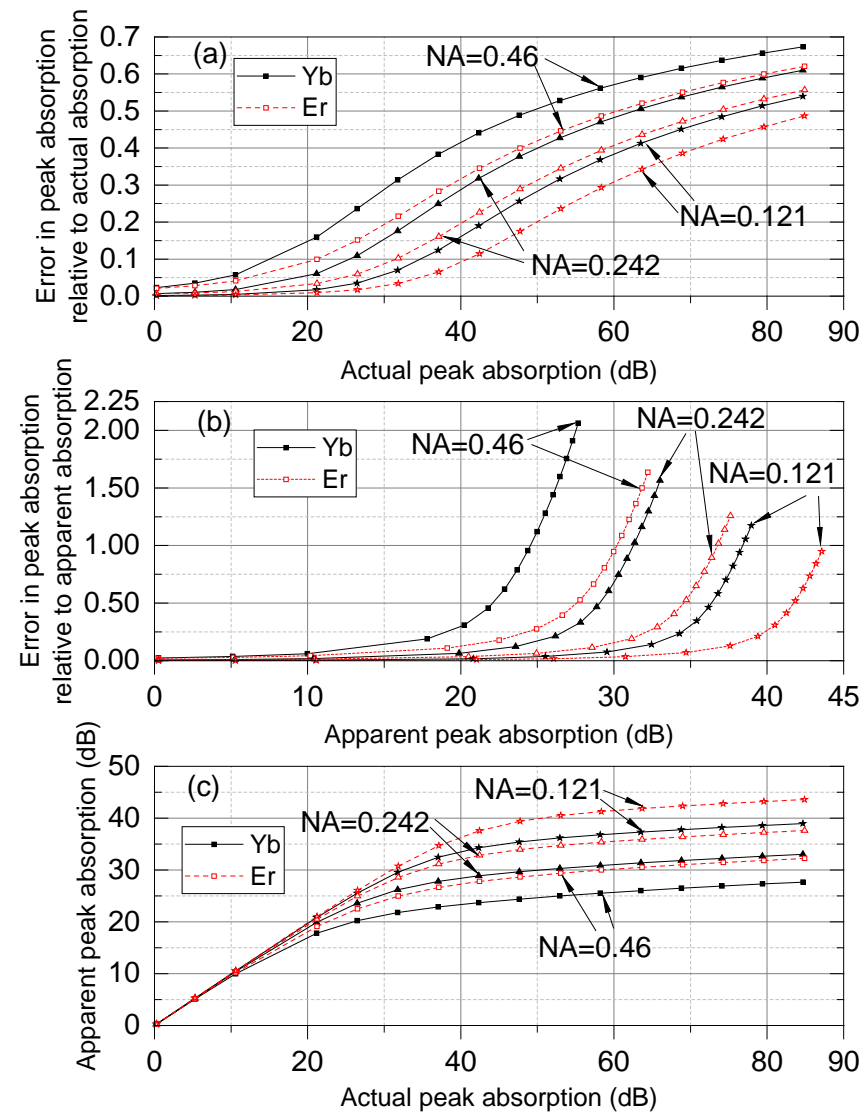

Fig. 8. (a) Error in peak absorption relative to actual peak absorption $((\alpha-$ $\left.\alpha_{w L S}\right) / \alpha$ ) vs. actual peak absorption $(\alpha)$, (b) error in peak absorption relative to apparent peak absorption $\left(\left(\alpha-\alpha_{W L S}\right) / \alpha_{W L S}\right)$ vs. apparent peak absorption $\left(\alpha_{W L S}\right)$, (c) apparent peak absorption $\left(\alpha_{W L S}\right) v s$. actual peak absorption $(\alpha)$ for Er and Yb-doped fibers of different NA and constant V-value $(V=2)$ with white-light source.

The errors are larger for the YDFs than for the EDFs. We attribute this to Yb's wider absorption spectrum and sharper peak (see Fig. 3). The long tail of the absorption spectrum allows for significant amounts of long-wavelength probe light to propagate with low loss before it is finally absorbed, while the sharp absorption peak ensures there is hardly any probe light left at the peak. The overlap of the emission peak with the absorption peak further increases the error at the peak, but since the spontaneous emission increases only linearly with the emission cross-section, the effect of this is modest. Simulations showed that the errors indeed become smaller if the spectrum is narrowed through truncation.

The dependence on the NA is explained by the larger fraction of re-emitted photons that a higher-NA core captures, with probability approximately equal to $N A^{2} /\left(4 n^{2}\right)$ into the forward-propagating mode [13]. An NA of 0.46 is unusual for a single-mode fiber, but 0.24 is quite common and still leads to large errors already at low absorptions. On the other hand, large-mode-area fibers can have an NA of, e.g., 0.06, which is lower than what is considered in Fig. 8. Re-emission effects will be reduced and often even negligible in practice in singlemode fibers with such low NAs, if all other parameters are equal.

\section{Low absorption regime}

Although the error increases for fibers with higher absorption, Fig. 8 shows an error also for short fibers. The 
source of this intriguing low-absorption error is still that absorbed photons are re-emitted and then recaptured by the forward mode and guided to the detector, but in contrast to the high-absorption regime, the error now increases at longer wavelengths, where the emission-to-absorption cross-section ratio is large. In order to illustrate this, Fig. 9 plots the fluorescence power $S_{A S E}$ as well as the loss in power in the probe, $S_{P}(\lambda, 0)-S_{P}(\lambda, L)$, for the YDF with an NA of 0.46 , with $0.5 \mathrm{~dB}$ peak absorption. It shows that the power loss in $S_{P}$ is partly compensated by the power generated in $S_{A S E}$. For some wavelengths this is particularly significant. In fact, with the parameters used in our simulations, the generated fluorescence power exceeds the power lost in the probe for wavelengths longer than $1064 \mathrm{~nm}$. This leads to a negative apparent absorption.

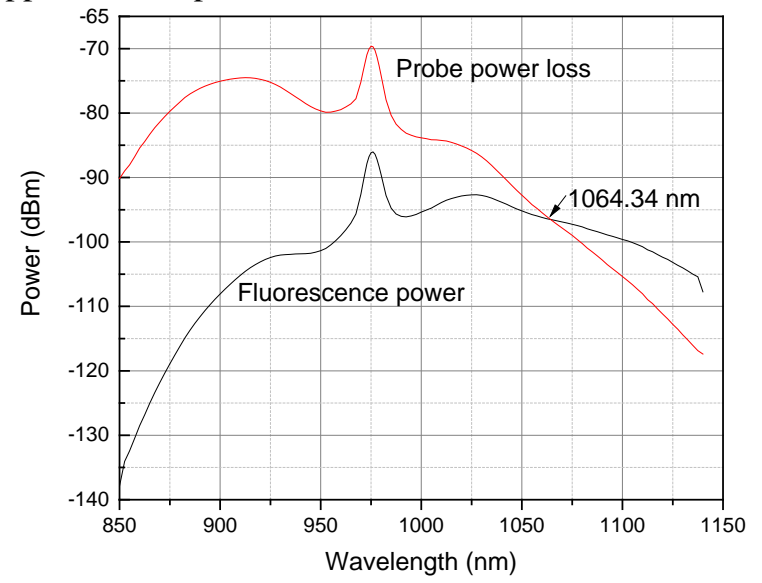

Fig. 9. WLS probe power loss as well as fluorescence power for YDF with NA of 0.46 , with $0.5 \mathrm{~dB}$ peak absorption.

A peak absorptions as low as $0.5 \mathrm{~dB}$ corresponds to a fiber length of only $12 \mathrm{~mm}$ with the $42-\mathrm{dB} / \mathrm{m}$ peak absorption we consider. Although they do not present any special difficulties, such low absorptions in such short fibers are seldom measured in practice. Nevertheless, it is certainly worth noting that with WLS-probing of high-NA fibers, the re-emission error cannot be eliminated completely. We conclude that in the presence of re-emission, alternative configurations or modeling combined with some fitting procedure is needed in order to accurately determine the absorption spectrum of a high-NA fiber, whether characterized in the low- or high-absorption regime.

\section{Simulations of tunable light source and broadband detection}

We next simulate the case with a tunable light source and broadband detection, i.e., with the photons wavelengthselected at the source rather than at the detector. According to the measurement data in Fig. 4, this also leads to significant errors. In this case, after initial absorption of TLS probe light, the captured re-emitted photons are distributed over the whole of the emission spectrum. These photons all register when using broadband detection, causing a reduction in the measured absorption. Fig. 10 shows simulation results for EDFs and YDFs. The errors are generally larger than those with WLS \& OSA, especially for Yb, although the difference is not dramatic. See comparison in Fig. 10(b).
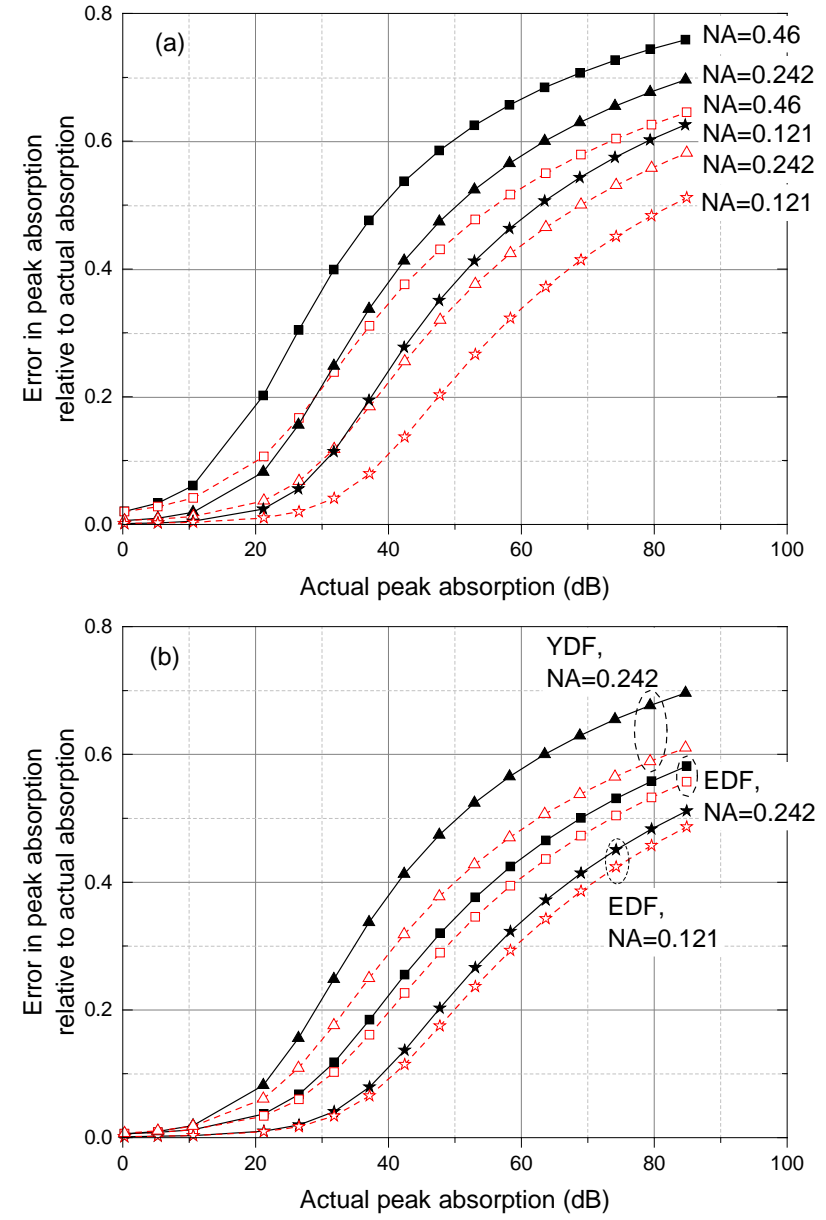

Fig. 10. (a) Error in peak absorption measured with TLS and broadband detection relative to actual peak absorption $\left(\left(\alpha-\alpha_{T L S}\right) / \alpha\right)$ for EDFs (red dotted curves with empty data markers) and YDFs (black solid curves with filled data markers) of different NA with $V=2$. (b) Selected curves from (a) (black solid curves with filled data markers) together with matching curves from Fig. 8 for WLS and OSA (red dotted curves with empty data markers).

Fig. 11 exemplifies output spectra and how the TLS probe and fluorescence powers change along the fiber. For short fibers the probe light dominates the output, but for long fibers the fluorescence power dominates and leads to lower measured absorption. This explains the errors in Fig. 10. The close-to straight line that is formed by the lowest-power parts of the spectra in long fibers is peculiar and can be understood as follows: According to (2), the spontaneous emission per unit fiber length and per unit wavelength into the guided mode is given by $2 h c^{2} \gamma_{e} \lambda^{-3}$. The wavelength-dependence of $\gamma_{e}$ is the same as that of $\sigma_{e}$, if we neglect the weak wavelengthdependence of the overlap integral in (3). Thus, the wavelength-dependence of the spontaneous emission follows $\sigma_{e} \lambda^{-3} \approx \sigma_{a} \exp \left[h c \Delta \lambda /\left(\lambda^{2} k_{B} T\right)\right] \lambda^{-3}$. Here, we have used McCumber's approximate relation [14] between $\sigma_{e}$ and $\sigma_{a}$, with $k_{B}$ being Boltzmann's constant, $T$ the temperature, and $\Delta \lambda$ the wavelength difference to the zero-phonon wavelength. Effectively, the fluorescence builds up over one absorption length. The absorption length is equal to $\left(N_{0} \Gamma \sigma_{a}\right)^{-1}$, so is inversely proportional to $\sigma_{a}$. Once the fluorescence dominates over the probe, the propagating spectral power per unit wavelength is proportional to the product of the two, i.e., $\sigma_{a}{ }^{-1}$ $\sigma_{e} \lambda^{-3} \approx \exp \left[h c \Delta \lambda /\left(\lambda^{2} k_{B} T\right)\right] \lambda^{-3}$. If we neglect the weaker $\lambda^{-}$ 3 -dependence then this will form a straight line on a 
logarithmic scale. Here we have also neglected the wavelength-dependence of the absorption overlap integral. In our regime of very weak pumping this is different from the overlap integral for $\gamma_{e}$ in (3), but again the wavelengthdependence is weak. We expect that a similar effect would show in Fig. 6, if longer fibers were included. In passing, we mention that if the wavelength-dependence of the spectrum by and large follows exp [ $\left.h c \Delta \lambda /\left(\lambda^{2} k_{B} T\right)\right] \lambda^{-3}$ then there is a simple integral expression for how $S_{A S E}$ evolves along the fiber. However we do not expect this will apply in any practical regime, given that the expression exp [hc $\Delta \lambda /\left(\lambda^{2} k_{B}\right.$ $T)] \lambda^{-3}$ is not valid for the peak of $S_{A S E}$.

Fig. 11(c) also plots the fluorescence (ASE) power for a simulation in which we truncated the propagating spectrum at $1580 \mathrm{~nm}$. The Er absorption and emission cross-sections are weak beyond $1580 \mathrm{~nm}$, but Fig. 11(a) shows that they will be important for $5 \mathrm{~m}$ of EDF. In this light, it is not surprising that the simulation with truncated spectrum leads to nearly $10 \mathrm{~dB}$ lower fluorescence after $5 \mathrm{~m}$ (105 dB peak absorption), and $3.75 \mathrm{~dB}$ lower after $2 \mathrm{~m}$ (42 dB peak absorption).
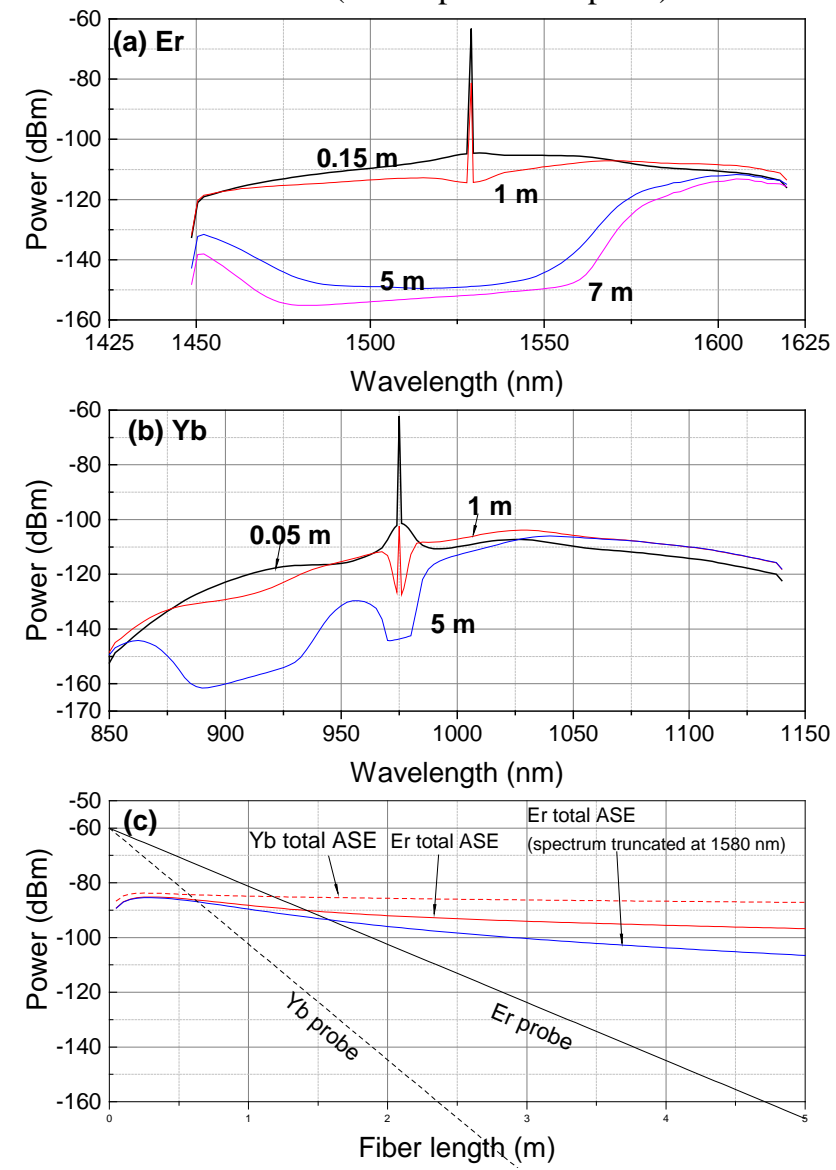

Fig. 11. Output spectra with TLS probe of (a) EDF with 0.242 NA from Fig. 10. (b) YDF with 0.242 NA. (c) Evolution of probe power and total fluorescence power along the fibers.

These results support the significance of a wide broad absorption shoulder for re-emission errors to occur. They also point to the importance of accurate cross-section spectra for accurate simulations at high levels of absorption. In our Ercalculations, the spectrum extended to $1619.8 \mathrm{~nm}$. Whereas the neglect of longer wavelengths has no impact on amplifier simulations, it may have a small effect on our absorption simulations. However we did not investigate this. For Yb- doped fibers, the simulations cover a larger fraction of the emission spectrum so we expect that the effect of truncating the simulations at $1140 \mathrm{~nm}$ is smaller.

\section{Simulations of tunable light source and optical spectrum analyzer}

Amongst the experimental data of Fig. 4, best is to use a TLS combined with an OSA, i.e., with spectral selection at both source and detector. To further investigate this configuration, we simulated how the error depends on actual peak absorption for the EDF with NA of 0.242. We find that the error is determined by the largest of the bandwidths at the source and the detector. A largest bandwidth of $0.1 \mathrm{~nm}$ allows us to measure $\sim 90 \mathrm{~dB}$ of absorption before re-emission incurs a $1 \%$ error in this fiber. See Fig. 12. Furthermore, in this configuration, the detected fluorescence power is proportional to the detected probe power (at fixed probe wavelength). This is in contrast to the case of WLS + OSA and TLS + broadband detector. It enables us to calculate the error for all filter bandwidth combinations (at fixed probe wavelength) from a single simulation, as long as the spectral variations over the filter bandwidths are small. Simulations confirmed that this is a reasonable assumption for bandwidths up to $1 \mathrm{~nm}$, and possibly more, in EDFs and YDFs, even at spectral peaks.

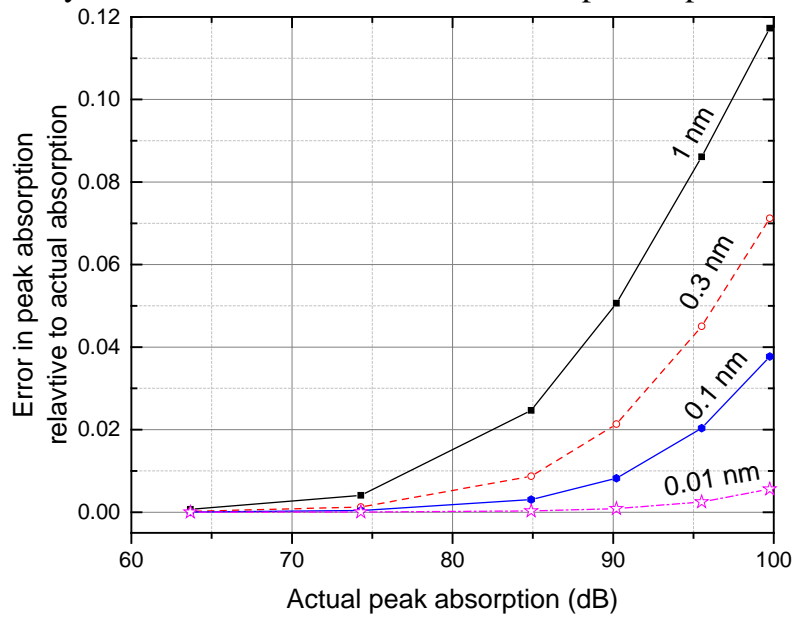

Fig. 12. Simulation of the relative measurement error $\left(\left(\alpha-\alpha_{W L S}\right) / \alpha\right)$ at the absorption peak for different largest value of source and detector bandwidth for EDF with NA of 0.242.

We expect that the TLS we used has a linewidth of the order of $1 \mathrm{fm}$. Thus the OSA bandwidth of $1 \mathrm{~nm}$ determines the effective bandwidth of our measurement in Fig. 4. According to Fig. 12, this allows us to measure $\sim 77 \mathrm{~dB}$ of absorption before re-emission errors reach $1 \%$. A smaller OSA bandwidth may allow us to measure even higher absorption, although the limits on launched power ( $100 \mu \mathrm{W}$ or preferably less) imposed by saturation in this fiber means that the detected power will be close to the noise floor of normal OSAs.

Several re-emission processes potentially contribute to the detected fluorescence power and thus to the error when a TLS is used together with an OSA. This includes processes with one, two, and three re-emission events. By comparing fibers with the same $\mathrm{V}$-value $(V=2)$ and actual absorption $(100 \mathrm{~dB})$ but different NAs $(0.121,0.242,0.3$ and 0.46$)$, we find that the detected fluorescence is approximately proportional to the fourth power of the NA. Since the probability of one photon to be captured by the guided mode is proportional to the square 
of the NA, this suggests processes with two re-emission events dominate. Specifically, a photon from the TLS (at the absorption peak) is absorbed and re-emitted at a lowabsorption wavelength. The photon propagates towards the detector, is absorbed again near the detector, and then reemitted at a wavelength within the receiver bandwidth. There could also be processes involving photons first re-emitted in the backward direction and then re-emitted again in the forward direction. However processes with backwardpropagating light would either have to involve a third reemission event (overall dependence proportional to the sixth power of the NA) or propagation over an extended distance at the peak absorption wavelength. Simulations confirmed that backward-propagating light had no influence.

There is no out-of-band light leakage in the simulations for Fig. 12. However single-emission events can also contribute to the error through finite of out-of-band suppression at source or detector. For example the TLS we used has an out-of-band level (in $1 \mathrm{~nm}$ ) which is approximately $40 \mathrm{~dB}$ below its total in-band power, and the total out-of-band power is $25 \mathrm{~dB}$ less than the in-band power. Some of this power is at low-loss wavelengths, so can propagate through the fiber and be absorbed and re-emitted at the peak. Simulations showed that this level does not significantly change the errors in Fig. 12 for up to $90 \mathrm{~dB}$ of absorption. Furthermore, the out-of-band level from our TLS is rather high, and it is straightforward to reduce it. There is also out-of-band leakage in the OSA, but this is expected to be much lower than in the TLS and can be neglected. Thus we conclude that it is possible to measure 90 $\mathrm{dB}$ Er-absorption before re-emission becomes a problem also with finite out-of-band extinction. Saturation and noise are then likely to be bigger concerns, so we conclude that the use of a TLS and an OSA can eliminate re-emission as a significant source of error in fibers with high absorptions.

We next consider the less common case of low absorption as in Fig. 9. Typically the absorption peak of a fiber has a width of more than $1 \mathrm{~nm}$. Thus, even if we only consider reemission of probe light absorbed by the peak, reducing the probe-light bandwidth to $0.01 \mathrm{~nm}$ will reduce the power of reemitted light by more than $20 \mathrm{~dB}$. Note that this is in a relative sense: Typically a narrow probe has a higher spectral power density and may even have a higher total power. However reemission is a linear process, and it is only the ratio of the inband to out-of-band power that matters, not the absolute values.

In Fig. 9, a 20-dB reduction of the re-emitted light ("fluorescence power") renders it negligible. Therefore, we conclude that also in the low-absorption regime, the use of narrow-band probe and detection can eliminate re-emission as a significant source of error.

Normally neither $0.5 \mathrm{~dB}$ nor $90 \mathrm{~dB}$ are best values to measure, if the experimentator is free to choose. Around 20 $\mathrm{dB}$ of absorption seems more appropriate, especially in case of a setup with spectral filtering in only one end of the fiber. However, it is not always straightforward or even possible to cut the fiber to $20 \mathrm{~dB}$ absorption. For example, Yb-doped fibers for cladding-pumping can have core-mode absorption of over $2000 \mathrm{~dB} / \mathrm{m}$. Although it is possible to work with $\mathrm{cm}$-long fibers, longer fibers are preferable, e.g., to improve the accuracy in cutback calculations. Then the absorption may approach $100 \mathrm{~dB}$. Although leakage of cladding-light makes it difficult to measure such high levels of core-mode absorption, it may still be possible with the techniques presented here and elsewhere [4], with efficient cladding-mode stripping and mode-selective coupling into as well as out from the core

\section{Additional modeling details}

We finally discuss some details of the modeling of special relevance to absorption simulations. The spontaneous emission into the guided mode is actually proportional to the product of the upper-state decay rate (given by the inverse of the fluorescence lifetime) and the upper-state population. Also in the standard model for a fiber amplifier that we used, the spontaneous emission into the guided mode is proportional to the upper-state population, but there is no explicit dependence on the decay rate. If the fluorescence lifetime used in simulations is too large then the upper-state population (proportional to the fluorescence lifetime according to (6)) becomes unphysically large, which implies that the radiative quantum efficiency exceeds unity. This is not necessarily critical for amplifier simulations, since it does not affect the relative strength of the fluorescence and gain and since stimulated emission typically dominates over spontaneous emission. Therefore, amplifier simulation software does not necessarily check the radiative quantum efficiency (RP Fiber Power does not). However for absorption simulations it is more important. Still, even a $1-\mathrm{dB}$ error in the radiative quantum efficiency would only have a small effect on the error in the absorption, in most cases. We used the variant of the Füchtbauer-Ladenburg equation adapted for large linewidths [15] to find the lifetime which results in unity quantum efficiency. The corresponding lifetimes are $0.902 \mathrm{~ms}$ for the YDF and $9.32 \mathrm{~ms}$ for the EDF, for NA $=0.242$. The lifetimes for other NAs is slightly different as we use a different refractive index for different NAs. Of course, the quantum efficiency can be lower than unity. This reduces the re-emission-induced absorption error, which vanishes completely for nonradiative systems.

A higher NA means that a larger fraction of the fluorescence is captured. However, the expression $N A^{2} /\left(4 n^{2}\right)$ for the capture ratio $\eta$ is only an approximation. For single mode fiber, when the density of excited ions $N_{2}$ follows the mode profile and we neglect any effect of the wavelength dependence of the mode profile, a more accurate expression is as follows.

$$
\eta=\frac{\lambda^{2}}{4 \pi n^{2}} \frac{\int_{0}^{b} I^{2}(r, \lambda) 2 \pi r d r}{\int_{0}^{b} I(r, \lambda) 2 \pi r d r}
$$

This expression follows from (2), (3), (5), and (6) combined with the well-known relation between the spectral power density of fluorescence and the emission cross-section spectrum. Fig. 13 shows how the capture ratio according to (11) depends on the V-value of step-index fibers with NA = $0.121\left(N A^{2} /\left(4 n^{2}\right)=0.0017\right)$ and NA $=0.242\left(N A^{2} /\left(4 n^{2}\right)=\right.$ 0.0069). Note that although the capture ratio remains 
proportional to $N A^{2}$ at fixed $\mathrm{V}$-value, the capture ratio for constant NA varies with the V-value, and $N A^{2} /\left(4 n^{2}\right)$ is $25 \%$ larger than (11) for $V=2$. The effect of the $V$-value on the reemission error can be assessed by scaling the fluorescence power $S_{A S E}$ according to Fig. 13. Fig. 14 compares the errors obtained with the WLS + OSA configuration for a 0.242-NA EDF of three different V-values. These as well as all our other simulations accurately accounted for the capture of fluorescence into the guided mode, as described by (2) - (6), i.e., in no case did we rely on the approximation $N A^{2} /\left(4 n^{2}\right)$.

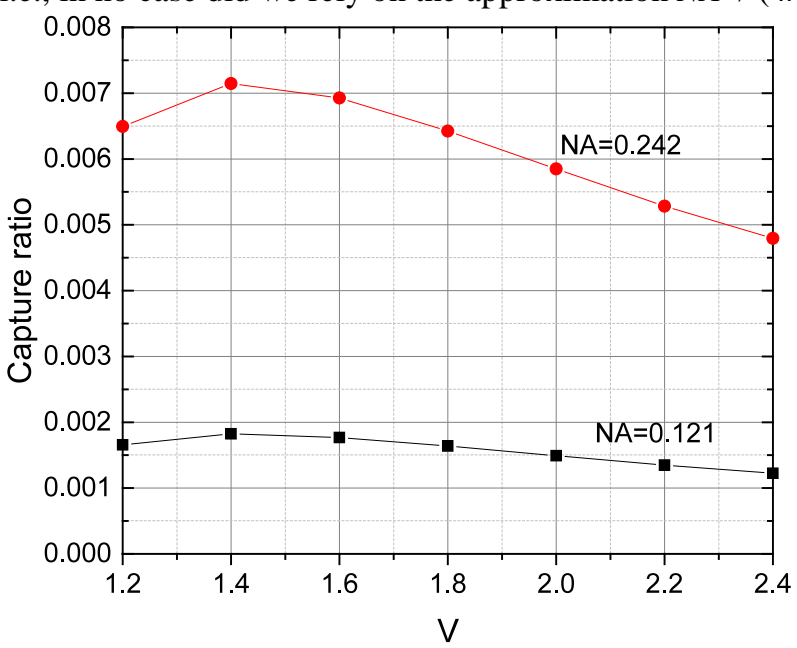

Fig. 13. Capture ratio according to (11) vs. V-value for NA of $0.121 \& 0.242$.

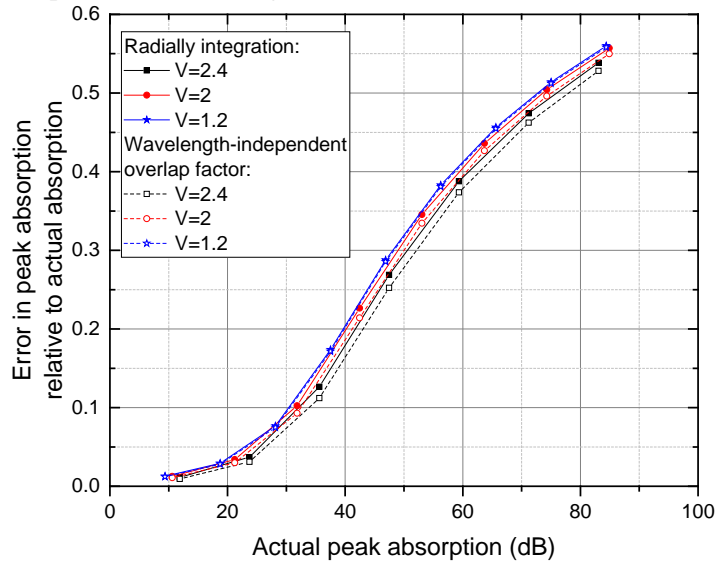

Fig. 14. Error in absorption relative to actual absorption $\left(\left(\alpha-\alpha_{W L S}\right) / \alpha\right)$ at absorption peak with the WLS + OSA configuration for an 0.242-NA EDF of three different $\mathrm{V}$-values obtained with radially resolved simulations (solid curves) and with wavelength-independent overlap factors (dotted curves).

A related point is the common use in amplifier simulations of wavelength-independent overlap factors in order to eliminate the need for repeated transverse integration according to (3) and (4). According to (1) and (2), this affects the evolution of $S_{P}$ and $S_{A S E}$ in a similar way. Nevertheless, analogously to the case of an unphysical quantum efficiency, the overlap approximation may be acceptable for amplifier simulations, but not necessarily for absorption simulations. Fig. 14 also compares the errors obtained with the radially resolved calculations that we used to those with wavelengthindependent overlap factors. The differences are small.

Absorption into high-lying levels can also influence the absorption measurements. $\mathrm{Er}^{3+}$ has a number of shorterwavelength absorption bands, and most photons absorbed by them would also be re-emitted into the $1.5-\mu \mathrm{m}$ band. This is a particular concern if the spectral power density is higher at shorter wavelengths. This was not included in our modeling, since we did not have any light at corresponding wavelengths. Similarly, the errors can also increase (or decrease) if there are variations in the spectral power density across the absorption band under study. These were $2 \mathrm{~dB}$ with our WLS, but were neglected in simulations.

\section{CONCLUSIONS}

Simulations as well as measurements show that spectrally overlapping re-emission can lead to unacceptable errors in the characterization of absorption spectra of single-mode fibers doped with erbium and ytterbium. The errors increase with the NA and with higher absorption values, and may become unacceptable even for $20 \mathrm{~dB}$ of absorption. Still, a smaller error can remain also in the limit of zero absorption. Reemission effects can be mitigated by the use of a narrow-line probe together with narrow-line detection. Although not analyzed in this paper, we note that it is also possible to use time-gating to preferentially select ballistic photons over reemitted ones. In the cw regime (and we expect also with modulated light), standard amplifier models work well for simulating absorption measurements, although one should ensure that the fluorescence lifetime is accurate so that the radiative quantum efficiency does not exceed unity. Even with simple light sources such as tungsten filaments, measurements at $20-30 \mathrm{~dB}$ of absorption may appear quite straightforward and accurate, and well above detector noise limits. It is then easy to be fooled by the apparent simplicity and accuracy of the measurements and overlook also significant re-emission errors. This is even more so with increasingly available supercontinuum sources.

We expect similar errors will occur for any dopant with spectrally overlapping emission and absorption. We also expect there can be errors also in geometries other than singlemode fibers, and multimode fibers are under investigation.

\section{ACKNOWLEDGMENT}

We thank Dr. Rüdiger Paschotta, Dr. Peter Moulton, Dr. Mathieu Laroche, Prof. Bill Brocklesby, \& Prof. David Hanna for fruitful discussions. All data supporting this study are openly available at http://dx.doi.org/10.5258/SOTON/402866.

\section{REFERENCES}

[1] I. D. Aggarwal, "Optical waveguide manufacturing," in Fiber Optics, James C. Daly ed. Boca Raton, FL, USA: CRC Press, 1984, pp. 21-50.

[2] Anping Liu and Kenichi Ueda, "The absorption characteristics of circular, offset, and rectangular double-clad fibers," Opt. Commun., vol. 132, no. 5-6, pp. 511-518, Dec. 1996.

[3] M. N. Zervas, and C. A. Codemard, "High Power Fiber Lasers: A Review,” IEEE J. Quantum Electron., vol. 20, no. 5, pp. 1-23, 2014.

[4] Anka Schwuchow, Sonja Unger, Sylvia Jetschke and Johannes Kirchhof, "Advanced attenuation and fluorescence measurement methods in the investigation of photodarkening and related properties of ytterbiumdoped fibers," Appl. Opt., vol. 53, no. 7, pp. 1466-1473, 2014.

[5] P. Koška, P. Peterka, J. Aubrecht, O. Podrazký, F. Todorov, M. Becker, Y. Baravets, P. Honzátko, and I. Kašík, "Enhanced pump absorption efficiency in coiled and twisted double-clad thulium-doped fibers," Opt. Express, vol. 24, pp. 102-107, 2016.

[6] C. A. Codemard, A. Malinowski, and M. N. Zervas, "Numerical optimisation of pump absorption in doped double-clad fiber with 
transverse and longitudinal perturbation,” in Proc. SPIE, vol. 10083, paper 1008315, 2017.

[7] R. W. Wood, "Scattering and Regular Reflection of Light by an Absorbing Gas," in Researches in physical optics, part II , New York, NY, USA: Columbia University Press, 1919, pp. 117-146.

[8] A. C. G. Mitchell and M. W. Zemansky, "Absorption lines and measurements of the lifetime of the resonance state,” in Resonance radiation and excited atoms, Cambridge, UK: Cambridge University Press, 1961, pp. 92-151.

[9] F. D. Colegrove, P. A. Franken, R. R. Lewis, and R. H. Sands, "Novel method of spectroscopy with applications to precision fine structure measurements,” Phys. Rev., vol. 3, no. 9, pp. 420-422, Nov. 1959.

[10] B. M. Zhang, J. Zhao, Y. Feng, S. Zhu, J. H. Price, and J. Nilsson, "Fiber Absorption Measurement Errors Resulting from Re-emission of Radiation, ” in ASSL, Berlin, Germany, 2015, pp. AM5A.20.

[11] Fiber-optic simulation and design software RP Fiber Power from RP Photonics, https://www.rp-photonics.com/fiberpower.html.

[12] R. Paschotta, J. Nilsson, A. C. Tropper and D. C. Hanna, "Ytterbiumdoped fiber amplifiers,” IEEE J. Quantum Electron., vol. 33, no. 7, pp. 1049-1056, July 1997.

[13] M. Morin, R. Larose, and F. Brunet, "Q-Switched Fiber Lasers,” in Rare-earth-doped fiber lasers and amplifiers, revised and expanded, Michel J. F. Digonnet ed. Boca Raton, FL, USA: CRC Press, 2001.

[14] D. E. McCumber, "Einstein relations connecting broadband emission and absorption spectra,” Phys. Rev., vol. 136, no. 4A, pp. A954-A957, Nov. 1964

[15] W. Krupke, "Induced-emission cross-sections in neodymium laser glasses,” IEEE J. Quantum Electron., vol. 10, no. 4, pp. 450-457, 1974.

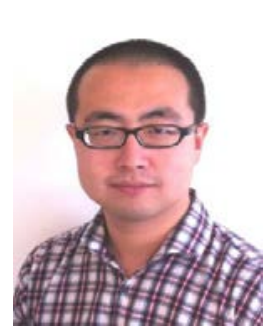

Yutong Feng received the B.S. degree in Applied Physics from Northwest University, Xi'an, China, in 2004 and the Ph.D. degree in Optical Engineering from Shanghai Institute of Optics and Fine Mechanics, Chinese Academy of Sciences, in 2009. From 2009 to 2012, he was a Research Assistant at North China Research Institute of Electro-Optics, Beijing, China. He is currently a postdoctoral research fellow at the Optoelectronics Research Centre (ORC), University of Southampton, UK. His research interests includes high power fiber lasers and amplifiers, nonlinear fiber optics, systems and applications.

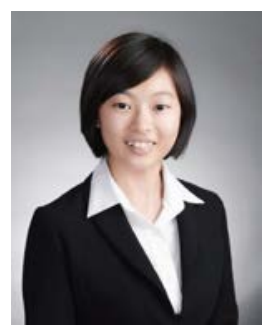

Betty Meng Zhang was born in Harbin, Heilongjiang, China in 1989. She received the B.E. degree in Electrical and Electronic Engineering from Nanyang Technological University, Singapore in 2013. She is currently pursuing the Ph.D. degree in Electrical and Electronic Engineering at Nanyang Technological University, Singapore, from 2015 to 2017 as a Visiting Academic with the ORC, University of Southampton, UK. Her research interest includes high energy ultrashort fiber laser systems and specialty optical fiber designs. Miss Zhang's awards and honors include Most Outstanding Student Paper Award (OFSIS 2017), Best Student Paper Award (OGC 2016), Women in Photonics Member of the Month (IPS Newsletter), IEEE Photonics Conference 2016 Women in Photonics Student Travel Grant (IPC 2016), IEEE Photonics Society Student Award (IPS).

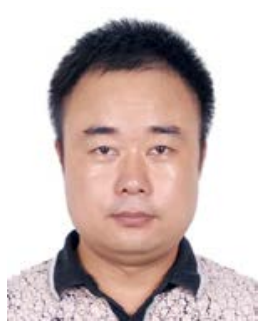

Junqing Zhao received the B.S. degree in Applied Physics from Henan Polytechnic University, China, in 2009 and the Ph.D. degree in Optical Engineering from Shenzhen University, China, in 2014. Then he started his postdoctoral research there and finished in Dec. 2016. From Jan. to Dec. 2015, as an academic visitor, he joined the High Power Fibre Lasers group at the ORC, University of Southampton, UK. Currently, he is working in the Jiangsu Key Laboratory of Advanced Laser Materials and Devices, School of Physics and Electronic Engineering, Jiangsu Normal University, Xuzhou, China. His research interests include pulsed fiber lasers, high power fiber lasers, mid-infrared laser generation via nonlinear optics technologies and components with active dopants, midinfrared supercontinuum generation, and related applications.

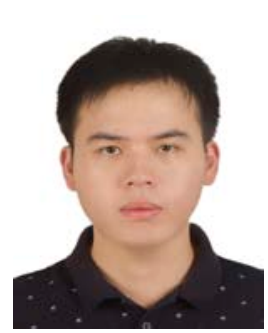

Sheng Zhu received the B.S. degree in Information Engineering from Shanghai Jiao Tong University, China in 2014 and M.Sc. degree in Photonics Technologies from University of Southampton, Southampton, UK in 2015. He is currently pursuing the Ph.D. degree at the ORC, University of Southampton. His research interests include high power fiber lasers

and amplifiers.

Jonathan H. V. Price received the Ph.D. degree in Optoelectronics from the ORC, University of Southampton, UK, in 2003. Subsequently, he was awarded a Royal Academy of Engineering Post-Doctoral Research Fellowship for his work at the ORC. His main research areas are applied nonlinear fiber optics using novel glass types and silica microstructured optical fibers for ultrashort-pulse wavelength conversion applications / supercontinuum generation and the development of high-power femtosecond pulsed fiber lasers.

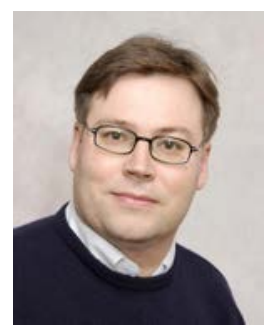

Johan Nilsson is a Professor at the ORC, University of Southampton, UK, and head of the High Power Fiber Lasers research group. In 1994, he received a doctorate in Engineering Science from the Royal Institute of Technology, Stockholm, Sweden, for research on optical amplification. Since then, he has worked on optical amplifiers and amplification in lightwave systems, optical communications, and guided-wave lasers, first at Samsung Electronics and later at ORC. His research has covered system, fabrication, and materials aspects, and in particular device aspects of high power fiber lasers and erbium-doped fiber amplifiers. He has published some 400 scientific articles. He is a fellow of the OSA and the SPIE, and a consultant to, and co-founder of, SPI Lasers. He is a member of the advisory board of the Journal of the Optical Society of Korea and was guest editor of two issues on highpower fiber lasers in IEEE Journal of Selected Topics in Quantum Electronics in 2009. He is a former chair of the Laser Science and Engineering technical group in OSA's Science and Engineering Council and is currently program 
chair for the EuroPhoton and Advanced Solid State Lasers conferences. 